\title{
BREAST DUCTAL COMPUTER PHANTOM
}

\author{
E. Franceschini, S. Mensah, D. Amy*, J.-P. Lefebvre
}

CNRS-LMA (Laboratoire de Mécanique et d'Acoustique), 31 Chemin Joseph Aiguier, 13402 Marseille Cedex 20, France

*University of Medecine Montpellier-Nîmes,UFR Kennedy, 30908 Nîmes Cedex 2, France

\begin{abstract}
Most of breast cancers (85\%) originate from the epithelium and develop first in the ductolobular structures. Therefore, the mammary epithelium should be investigated in priority by means of an anatomically guided examination. For this purpose (mass screening, surgery navigation), we develop a two-dimensional anatomic phantom which corresponds to an axial cut of the ductolobular structures and enables one to better understand the interaction of the breast composition with ultrasound. The different constitutive tissues are modeled as a random inhomogeneous continuum with density and sound speed fluctuations. Ultrasonic pulse propagation through the breast computer phantom has been simulated using a finite element time domain method (the phantom can be used with others propagation codes). The simulated Ductal Echographic image is compared with the Ductal Tomographic (DT) reconstruction. The preliminary results reveal a higher quality of DT both in terms of contrast and resolution.
\end{abstract}

Key words: Diffraction tomography, Ductal Echography, breast model, MFEM, near-field.

\section{NEED FOR AN ANATOMICALLY-LED METHOD OF INVESTIGATION}

Breast cancer and all the specific diseases of the breast present the common property of originating from the epithelium and developing first in the ductolobular structures. Therefore, the observation of these structures provides direct means for detecting alterations induced by breast diseases as soon as they become perceptible. Unfortunately, X-ray mammography does not visualize epithelium which is radio transparent. Magnetic Resonance Imaging (MRI) and echography are the two techniques which permit to visualize the three breast tissue constituents (the connective tissue, the fat and the ductolobular structure). MRI is increasingly employed mainly for breast examinations of young women. However, today, the resolution is still insufficient and this method of investigation remains limited for economic and technical reasons. Echography is well adapted to soft tissue examinations but, in conventional echography, transductal scans are performed perpendicularly to the ductal course. This mode of scanning cannot match the radial arrangement of breast anatomy. Cancers are detected only when they show an abnormality with a contrast and have a sufficient three dimensional volume, perceptible whatever the sweeping 
orientation ( $5 \mathrm{~mm}$ size lesions). The lack of anatomic guidance in breast ultrasound has been responsible for its failure [1][2].

An anatomically led method of investigation based on the identification of internal mammary structures, Ductal Echography (DE), was introduced in 1987. This technique is based on a radial scanning. Ductal-axial scans are performed along the ductal course. With this new technique, the observer investigates the epithelial structures actively by systematically following the ductal system in each mammary lobe. Figure 1 shows a DE and its interpretation.
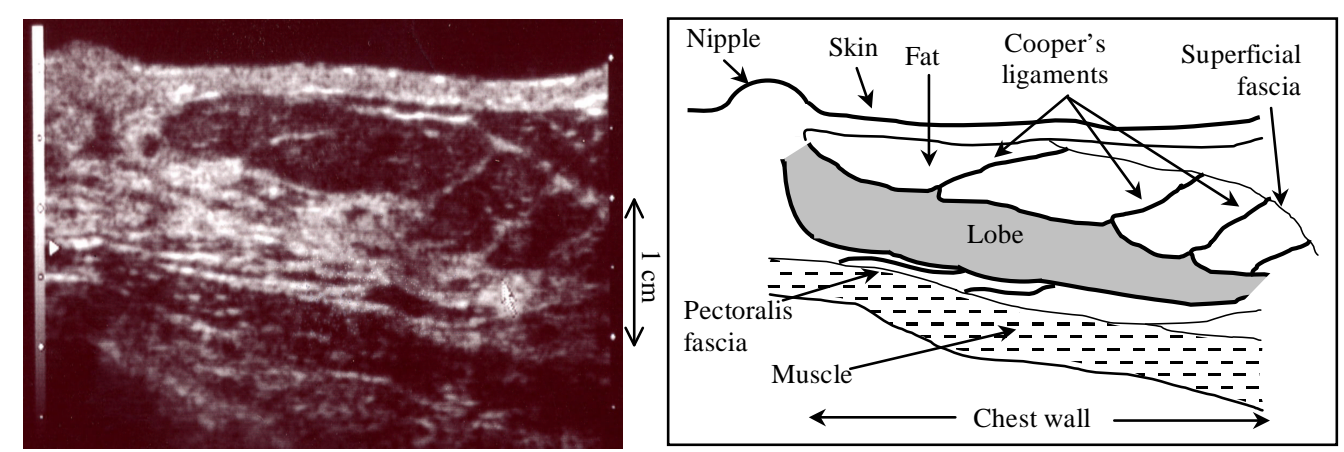

Figure 1: Ductal Echography. Typical aspect of a lobe totally displayed from the nipple to the periphery of the breast.

Our research aims at developing ultrasound tomography techniques that will reduce the operator-dependency observed in echography and provide an intelligible visualization of the epithelium. Indeed, tomographic methods in medical ultrasound are the subject of increasing interest. Current research work aims mainly at improving the differentiation of breast tissues (and cancers) by quantifying the sound speed, the elasticity and the attenuation of tissues [3] [4] [5] [6]. We propose to differentiate the breast tissues by using not only these parameters but also the breast anatomy identification as performed in DE.

In order to compare DE and our tomographic approach [7], ultrasonic pulse propagation through breast tissues has been simulated using a 2D realistic breast phantom that respects the anatomy of the breast as much as possible. The tissues of the breast are modeled as a random inhomogeneous continuum [8] [9]. For the echography, that serves as a common reference, we simulate a linear probe, a semi-circular antenna is used for the tomography. Tomographic and echographic reconstructions can then be compared.

\section{NUMERICAL PHANTOM}

\subsection{Wave propagation modeling}

The numerical method we use to compute the solution of the wave equation has been developed by C. Tsogka. This method models the time domain acoustic wave propagation in fluid media and is based on the discretization of the mixed velocity-pressure formulation for acoustics. The space discretization of the problem is based on a mixed finite element method [10] and for the 
discretization in time, a $2^{\text {nd }}$ order-centered finite difference scheme is employed. The simulation grid is surrounded with an absorbing layer (PML) for simulating unbounded domains [11].

This numerical method models the breast as a fluid of variable velocity and density. The experimental results of Frizzel's and Madsen's groups [12] [13] demonstrate that, in soft tissues, the absorption of shear waves is much greater than the absorption of pressure waves. This enables us to adopt an acoustic model at the frequencies (1-5 MHz) used.

\subsection{Tissue random media}

The numerical tissue-like materials are characterized in terms of density and celerity. We assume each parameter to consist of a heterogeneous mean value and the realization of a stationary random medium. The differences between the material parameters and their mean values are defined by correlation functions assumed to be isotropic. This assumption is not realistic, but locally, it is acceptable. Nevertheless, large scale fluctuations within each kind of tissue (connective tissue, lobe, fat) should also be considered, but they are not introduced in our models. Thus, we realize the stationary random medium in terms of a white noise filtered by a spectral filter, whose power spectrum is the radial Fourier Transform of the correlation function [8] [9].

A Gaussian filter is used since it produces tissue-like echographic patterns. It allows us to adjust the correlation length, and is quite simple to use. It leads to a correlation function of the type ( $a$ is the Gaussian correlation length):

$$
c(\underline{y})=\frac{\alpha}{\pi a^{2}} \cdot e^{-\left(\frac{y}{a}\right)^{2}}
$$

\section{3 "Realistic" numerical phantom}

The density and sound speed grids used for the computation were obtained by mapping a schematic view of a diametric section of the breast passing through the nipple. The drawings [figures 2 and 3] display the successive elements observed in the breast with ultrasound: the skin, the fat (including the superficial fatty layer and the deep fatty layer), the lobe, the connective tissue which supports the lobe and the intra-lobular structures. Cooper's ligaments link the lobes to the superficial layer of the superficial fascia lining the skin, and to the deep layer of the superficial fascia that lines the thoracic wall [1].

Each pixel of the map is then assigned a sound speed and density value determined from the results found in the relevant literature and assumed to be representative of the local tissue type. The water sound speed and density are those of water at $25^{\circ} \mathrm{C}$. The skin and the connective tissue sound speed employed were found respectively in [4] and [6]. Sound speeds for fat, glandular tissue and pectoralis muscle were obtained by the average values of human tissues given in [3] and [4]. Sound speeds for the ducto-lobular tree in healthy and pathological cases were determined from [3] and [5]. Density values for the tissues were determined from measurements summarized in [14] by the average values respectively reported for adipose tissue (or fat), connective tissue, mammary gland, skeletal muscle, and skin. 
Random media, defining the standard deviation and the correlation length for each tissue, are realized thanks to a DE analysis. Figure 1 displays several successive layers of tissues differentiated by their different echogenicities: the skin, an echogenic uniform $3 \mathrm{~mm}$ superficial layer; the superficial fascia, a more echogenic line close to the skin; the anterior fatty layer, an area of low echogenicity; the superficial Cooper's ligaments which appear bright when perpendicular to the probe and lose echogenicity when oblique to the probe; the lobe, an oblong echogenic layer; the deep fatty layer, an area of low echogenicity; the deep Cooper's ligament; the pectoralis fascia, a bright thin line covering the muscle layer; the muscle layer, an hypoechogenic striated aspect; the chest wall, a hypoechogenic muscular strip [1]. Consequently, the correlation lengths (and respectively the standard deviations) of the connective tissue (fasciae and Cooper's ligament), the hyper echogenic skin and the lobe are chosen smaller (respectively bigger) than the fat and the pectoralis muscle. We display a duct infiltration (ectasia) which has a fine texture; its correlation length is the smallest. The parameter values employed in the present study are summarized in Table 1 .

The finite element code, described in [paragraph 2.1], was employed to compute the propagation of cylindrical waves (2D simulations) through the phantom. The spatial step size of the finite element grid was chosen to be one fortieth of the wavelength. Each simulation was performed on IDRIS (Institute for Development and Resources in Intensive Scientific computing) using a Compaq Linux Cluster incorporating 24 Alpha EV68 processors cadenced at $836 \mathrm{MHz}(1.5$ Gigaflop peak performance per processor).

\begin{tabular}{|c|c|c|c|c|}
\hline \multirow{2}{*}{ Medium } & \multicolumn{3}{|c|}{ Average } & \multicolumn{2}{c|}{ Random medium } \\
\cline { 2 - 5 } & $\begin{array}{c}\text { Sound speed } \\
(\mathrm{m} / \mathrm{s})\end{array}$ & $\begin{array}{c}\text { Density } \\
\left(\mathrm{kg} / \mathrm{m}^{3}\right)\end{array}$ & $\begin{array}{c}\text { Standard } \\
\text { deviation } \\
(\%)\end{array}$ & $\begin{array}{c}\text { Correlation } \\
\text { length } \\
(\mathrm{mm})\end{array}$ \\
\hline Water & 1500 & 1000 & - & - \\
\hline Skin & 1590 & 1090 & 2 & 0.21 \\
\hline Connective tissue & 1545 & 1090 & 2 & 0.21 \\
\hline Fat & 1470 & 950 & - & - \\
\hline Lobe & 1550 & 1060 & 2 & 0.21 \\
\hline Ectasia & $1570-1620$ & 1040 & 0.8 & 0.02 \\
\hline Pectoral is muscle & 1545 & 1050 & 0.8 & 0.42 \\
\hline
\end{tabular}

Numerical breast phantom for echography. During a clinical examination, the patient lies on her back, her breast is flattened; a water bag is placed on the probe, thus the skin appears quite rectilinear. For the Finite Element Code, a grid of $2400 \times 2600$ pixels $(\Delta x=0.0075 \mathrm{~mm}$, $1.8 \mathrm{~cm} \times 1.95 \mathrm{~cm}$ ) was employed. Signals were recorded by 168 receivers simulating a linear probe placed about $4.5 \mathrm{~mm}$ from the skin surface. The computation time for the whole simulation was of the order of 1340 hours. Figure 2 represents the sound speed and density maps for the pathological situation corresponding to an ectasia. The size of this lobe corresponds to the lower lobes which are the smallest.

Numerical breast phantom for tomography. During an ultrasound examination, the patient lies prone on an examination bed, her breast immersed in a water bath, with a semi-circular antenna around it. For the Finite Element Code, a grid of $2400 \times 1200$ pixels $(\Delta x=0.015 \mathrm{~mm}, 3.6 \mathrm{~cm} \times 1.8 \mathrm{~cm})$ was employed. Short pulses were transmitted along the acquisition circle at every $0.25^{\circ}$ ( 720 shots). 
For each shot, numerical signals were registered by means of receivers over a finite aperture of $20^{\circ}$ centred on the transmitter, with a step of $0.25^{\circ}$. The computation time for the whole simulation was of the order of 1440 hours. Figure 3 represents the sound speed map for a diametric section of the breast passing through the nipple for a healthy situation (right lobe) and a pathological situation (left lobe).


Figure 2 : Numerical breast phantom for echography. Sound speed and density maps



Figure 3: Numerical breast phantom for tomography. Sound speed map (mm).

\section{IMAGING TECHNIQUES}

\subsection{Diffraction Tomographic reconstruction}

With the aim of solving the inverse problem, i.e. starting from measurements of the scattered field to recover the various parameter maps, one has to solve the forward problem (prediction). For that purpose, soft biological tissues are classically described by their density and their compressibility distributions. Provided the receiving aperture is large enough, this formulation has the advantage of leading to a single parameter reconstruction which corresponds to the Laplacian of the compressibility map [15]. This allows us (linearity of the problem) to neglect right now the density echo contributions, under the condition we limit our reconstruction procedure to the large aperture reflection tomography. Let $\gamma_{\chi}=\left(\chi-\chi_{0}\right) / \chi_{0}$ be the relative compressibility fluctuations, we can then introduce the forward problem. 
Forward problem. Let us consider a pressure source in the infinite homogeneous host medium, the scattered field $p_{d}$ meets the classical wave equation based on the assumption that the total field inside the region of interest (ROI) is very close to the incident field (first order Born approximation),

$$
-\frac{1}{c_{0}^{2}} \frac{\partial^{2} p_{d}}{\partial t^{2}}+\nabla^{2} p_{d}=\frac{\gamma_{\chi}}{c_{0}^{2}} \frac{\partial^{2} p_{i}}{\partial t^{2}}
$$

This equation can be resolved by the 3D Green function. We assume that the medium is excited by a spherical wave of infinite bandwidth (short pulse), transmitted from a point source $\underline{x}_{E}$, and measured at the receiver location $\underline{x}_{R}$. In order to simplify the formulation, we can introduce a new parameter:

$$
\gamma(\underline{x})=\frac{\gamma x(\underline{x})}{16 \pi^{2} c_{0}^{2}\left|\underline{x}_{R}-\underline{x} \| \underline{x}-\underline{x}_{E}\right|}
$$

Therefore, the scattered field is:

$$
\left.p_{d}\left(\underline{x}_{E}, \underline{x}_{R}, t\right)=\int_{\mathcal{R}^{3}} \mid \delta\left(\left|\underline{x}^{\prime}-\underline{x}_{E}\right|+\left|\underline{x}_{R}-\underline{x}^{\prime}\right|-c_{0} t\right) * f^{\prime \prime}(t)\right] \cdot \cdot\left(\underline{x} \underline{x}^{\prime}\right) d \underline{x}^{\prime}
$$

where $f^{\prime \prime}(t)$ is the second derivative of the wave form function $f(t)$.

We note here, that for a fixed time delay $t$ and a given acquisition geometry $\left(\underline{x}_{E}, \underline{x}_{R}\right)$, the locus of the point for which the argument of the delta function is zero is the ellipsoidal surface described by the equation $c_{0} t=\left|\underline{x}-\underline{x}_{E}\right|+\left|\underline{x}_{R}-\underline{x}\right|$.

Considering this acquisition geometry, the amplitude of the scattered field results from the integral of the compressibility distribution over the surface of the ellipsoid of revolution. This is why we use a qualitative algorithm, presented in [7], based on elliptical back-projection which deals with these data in order to recover the 2D distribution of $\chi(\underline{x})$.

\subsection{Echographic reconstruction}

Recent commercial ultrasound systems offer various techniques for breast investigation. The usual ultrasonic imaging mode, the B-scan (brightness-scan), consists in representing signal strength as brightness of a point.

The basic assumptions for an image formation are first, straight-line propagation; and secondly, constant speed of sound. Under these assumptions, the origin of each echo is calculated from its arrival time. Linear array transducers are generally used in breast imaging. A symmetrical subset of elements forms the working aperture, each active element of this aperture transmits a pulse according to a standard phasing rule leading to point focusing. The principle is the same for reception. One line of reflectivity is built up by considering several focal segments. A subsequent shift of the aperture results in an adjacent line of information. The final result is a rectangular image, made up of many (up to 256) lines.

We simulate a 168 element transducers with a central frequency of $5 \mathrm{MHz}$, using an aperture with 12 active elements. The piezoelectric transducers are represented by punctual sources (used 
both in emission and reception), and are spaced evenly at $\lambda / 3$. Each punctual source transmits a cylindrical wave (2D simulation). We use transmission and reception focusing at $6.3 \mathrm{~mm}, 8.85 \mathrm{~mm}$, $11.4 \mathrm{~mm}, 13.95 \mathrm{~mm}$ and $19.05 \mathrm{~mm}$, offering a dynamic range scanning from $5 \mathrm{~mm}$ up to $20.3 \mathrm{~mm}$.

\section{RESULTS}

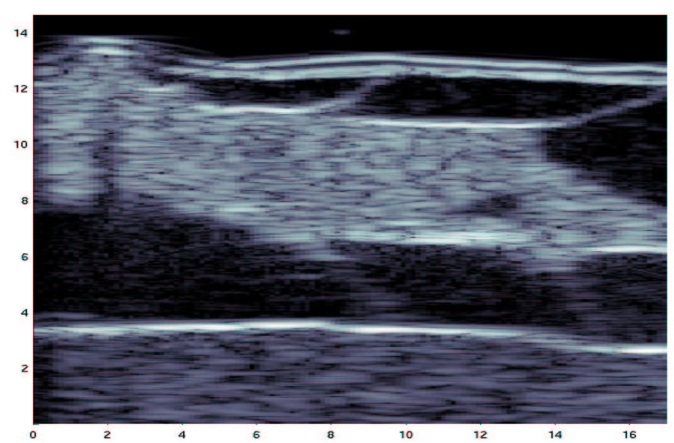

Figure 4: Echographic simulation [2.5-7.5 MHz], linear scanning (mm).


Figure 5: Numerical phantom and tomographic reconstruction [1.5-4.5 MHz].

The simulated echographic view [figure 4] has to be compared with the phantom of figure 2 . One may observe that the main features (lobe, ligaments, fat and retro-areola zone) are restituted. The lesion is visible, but not the fatty inclusions in the lobe. The speckle noise is important as it is expected from low frequency echography.

In the Tomographic Image (TI) [figure 5], all the interfaces (connective tissues) are clearly revealed; some intra-lobular fatty zones visible in TI are not restituted by DE. The TI is free of speckle even if a reconstruction noise is still noticeable. Compared with DE, the contrast in TI is greatly improved. In addition the border of the lesion is nicely delineated although the central frequency of the tomographic signals is half that of the echographic pulses. In both cases, the ducts are not visible, since the impedance contrast is weak and their size smaller than the wavelength.

The use of lower frequency responds to the computing limitation trade- off between resolution and acceptable computing time. Indeed, in comparison with the flattened breast, the uncompressed 
breast occupies an apparently larger volume. In spite of this low frequency activation, the image resolution is improved, which augurs new possibilities for current working frequencies comprised between [5-10 MHz].

\section{CONCLUSION}

This work focuses on the design of a 2D numerical acoustic model of the breast, making it possible to simulate ultrasonic wave propagation and measurement. The main features of this phantom are, on the one hand, its taking into account of anatomic details of sub-millimeter size and on the other hand, the respect of the ultrasonic characteristics of the various types of tissues (lobe, connective tissues, fat, muscle) constituting the breast which are expressed in terms of density and celerity maps.

Nevertheless, this phantom still presents two major limitations. Firstly, the acoustic model neglects the attenuation phenomenon which is of prime importance, especially as far as breast ultrasound semiology is concerned. Secondly, it is a 2D phantom that cannot permit full 3D measurements of the scattered field. Concerning the first item, our team is currently working on a visco-acoustic extension of the Finite-Element code by introducing a complex, frequency dependent visco-acoustic modulus (constant quality factor Q). Besides, signal pre-processing is required upstream the tomographic reconstruction in order to extract from the ultrasonic data, the integral attenuation along the propagation paths. The second point is more difficult to solve. Indeed, nowadays, 3D simulations seem to be beyond the capacity of middle of the range computers (computation time). Nevertheless, this 2D breast phantom enables us to simulate 2D Ductal Echographic images by using [2.5-7.5] MHz linear array probes. These images have been compared with the large aperture tomographies. In the latter case, [1.5-4.5] $\mathrm{MHz}$ cylindrical waves were transmitted and measured by point transducers uniformly distributed on a semi-circular antenna. The comparison of these images leads to a comfortable superiority of the tomographic reconstruction both in terms of contrast and in resolution. In addition, tomography will open the way to quantitative imaging that means, to computer assisted diagnoses. This is the next challenge of ductal ultrasound computed tomography and a push for realistic breast phantom designing and numerical experimentation based on clinical data .

\section{ACKNOWLEDGEMENT}

This study has been supported by Ville de Marseille, Region Provence-Alpes-Côte d'Azur, CNRS-LMA, and an "Action Concertée Incitative" of the French Ministry of Research and Technology (2001-2004). We are grateful to Veronique Bourrillon (ENSPM) for her participation in the echographic reconstruction developments.

\section{REFERENCES}

[1] Teboul M., Practical ductal echography, guide to intelligent and intelligible ultrasonic imaging of the breast (editorial Medgen, 2004). 
[2] Amy D., Critères échographiques de bénignité, in Echographie mammaire, De l'image à la thérapeutique (Collection d'imagerie radiologique, Ed. Masson, 1998), pp. 23-31.

[3] Kossoff G., Fry E. K., Jellins J., Average velocity of ultrasound in the human female breast , J. Acoust. Soc. Am. 53(6), pp.1730-1736 (1973).

[4] Goss S. A., Johnston R. L., Dunn F., Comprehensive compilation of empirical ultrasonic properties of mammalian tissues, J. Acoust. Soc. Am. 64(2), pp.423-457 (1978).

[5] Glover G. H., Characterization of in vivo breast tissue by ultrasound time of flight computed tomography, Ultrasonic tissue characterization II, M. Linzer, Ed. National Bureau of Standards Spec. Pub. 525, pp.221-225 (1979).

[6] Foster F. S., Strban M., Austin G., The ultrasound macroscope: initial studies of breast tissue, Ultrasonic imaging 6, pp.243-261 (1984).

[7] Mensah S., Ferriere R., Near field diffraction tomography, Ultrasonic Imaging 24, pp.135-146 (2002).

[8] Insana M. F., Wagner R. F., Brown D. G., Hall T. J., Describing small-scale structure in random media using pulseecho ultrasound, J. Acoust. Soc. Am. 87(1), pp.179-192 (1990).

[9] Klimes L., Correlation functions of random media, Pure and Applied Geophysics, 159 (2002).

[10] Bécache E., Joly P., Tsogka C. (2000), An analysis of new mixed finite elements for the approximation of wave propagation problems, SIAM J. Numer. Anal. 37(4), pp.1053-1084.

[11] Collino F., Tsogka C., Application of the pml absorbing layer model to the linear elastodynamic problem in anisotropic heteregeous media, Geogysics 66, pp.294-305 (2001).

[12] Frizzell L. A., Carstensen E. L., Shear properties of mammalian tissues at low megahertz frequencies, J. Acoust. Soc. Am. 60(6), pp. 1409-1411 (1976).

[13] Madsen E. L., Sathoff H. J., Ultrasonic shear wave properties of soft tissues and tissuelike materials, J. Acoust. Soc. Am. 74(5), pp. 1346-1355 (1983).

[14] Woodard H. Q., White D. R. , The composition of body tissues, The British Journal of Radiology 59, pp.1209-1219 (1986).

[15] Mensah S., Lefebvre J.-P., Enhanced Compressibility Tomography, IEEE Trans. Ultras. Ferro. Freq. Contr. 44(66), pp.1245-1252 (1997). 